\title{
La guérilla espagnole dans la guerre contre l'armée napoléonienne
}

\section{Vittorio Scotti Douglas}

\section{(2) OpenEdition \\ 1 Journals}

\section{Édition électronique}

URL : https://journals.openedition.org/ahrf/1683

DOI : 10.4000/ahrf.1683

ISSN : 1952-403X

Éditeur :

Armand Colin, Société des études robespierristes

\section{Édition imprimée}

Date de publication : 1 juin 2004

Pagination : 91-105

ISSN : 0003-4436

\section{Référence électronique}

Vittorio Scotti Douglas, "La guérilla espagnole dans la querre contre l'armée napoléonienne », Annales historiques de la Révolution française [En ligne], 336 | avril-juin 2004, mis en ligne le 15 juillet 2007, consulté le 22 avril 2022. URL : http://journals.openedition.org/ahrf/1683 ; DOI : https://doi.org/ 10.4000/ahrf.1683

Ce document a été généré automatiquement le 22 avril 2022.

Tous droits réservés 


\title{
La guérilla espagnole dans la guerre contre l'armée napoléonienne ${ }^{1}$
}

\author{
Vittorio Scotti Douglas
}

Le mot et son histoire

1 Le terme guérilla est entré aujourd'hui dans le langage commun en plusieurs langues: guerrilla warfare en anglais, guerriglia en italien, Guerrillakrieg ou simplement Guerrilla en allemand, guerrilha en portugais, langue qui toutefois emploie aussi le terme guerra subversiva ${ }^{2}$. La signification attribuée au mot est -par convention- désormais reconnue et acceptée partout. La définition la plus complète à ma connaissance parle de «lutte illégale d'organisations ou groupes non autorisés à des opérations militaires, contre le pouvoir légitime de l'État ou contre une puissance d'occupation $»^{3}$. De toute évidence cette définition est rigoureusement juridique, puisqu'en effet, tout groupe de civils armés qui s'adonne à des opérations militaires agit de manière «illégale ». Ce n'est pas le lieu d'entamer ici une discussion concernant l'absence de reconnaissance de la guérilla par les conventions de guerre, même les plus récentes.

2 Un texte célèbre -Guerrilla-, rédigé par un homme qui appliqua avec intelligence et hardiesse ses théories sur le terrain, est celui de T. E. Lawrence, pour la quatorzième édition de l'Encyclopaedia Britannica (1929). Le texte était précédé d'un premier paragraphe analytique, signé par Sir Thomas Barclay, vice-président de l'International Law Association ${ }^{4}$.

3 Mais d'où vient ce mot à consonance espagnole, et surtout pourquoi l'emploie-t-on aujourd'hui dans l'acception que l'on vient de citer? Il s'agit de l'adaptation française du diminutif espagnol guerrilla (petite guerre) du terme guerra, qui n'a pas besoin de traduction. En espagnol, le mot guerrilla est défini pour la première fois en 1611, dans le célèbre dictionnaire de Covarrubias 5 . Plus tard, dans les différentes éditions (1734, 1780, 1783 et 1791) du Diccionario de la Real Academia de España, le terme signifie «encuentro ligero de armas» (choc léger d'armes), «contrariedad de dictámenes de poca entidad » (contrariété d'opinions, ou de rapports, de faible importance), et on cite aussi un jeu de cartes nommé guerrilla. Comme diminutif du mot guerre, il fut employé pour traduire en espagnol le fameux ouvrage de Grandmaison, La petite guerre ou traité du 
service des troupes légères en campagne, certainement le plus connu des nombreux traités parus en la matière ${ }^{6}$ dans la seconde moitié du XVIIIe siècle ${ }^{7}$. L'ouvrage jouit d'une fortune aussi démesurée que surprenante, et devint très rapidement un texte de référence, cité et traduit partout ${ }^{8}$. La première traduction espagnole fut publiée en 1780, sous le titre La Guerrilla ó Tratado del servicio de las Tropas ligeras en Campaña ${ }^{9}$. De là vient l'équivalence entre guerrilla et petite guerre, et l'expression partidas de guerrilla fut employée pour désigner les petits détachements d'infanterie destinés aux attaques par surprise ou aux actions de reconnaissance. On faisait donc référence à des opérations de troupes légères régulières, ordinairement en petites formations, homologues alors des partis français, ou des partite italiennes ${ }^{10}$. Les deux termes furent utilisés avec cette signification au début de la Guerra de la Independencia, comme dans le bulletin du Général Castaños, après la bataille de Bailén, le 27 juillet $1808^{11}$, comme aussi dans des écrits de 1814, qu'il s'agisse d'une biographie de l'Empecinado ${ }^{12}$, ou de Mémoires contemporains restés longtemps inédits ${ }^{13}$. On peut donc dire qu'en 1808 , au début de la guerre en Espagne, le terme guerrilla indiquait des opérations militaires secondaires, et l'on continua encore à l'employer dans ce sens pendant un certain temps. Cependant l'usage en était de plus en plus répandu, et entrait avec force dans les autres langues, avec la signification moderne de lutte armée de civils encadrés en formations irrégulières, contre un ennemi envahisseur et aussi, comme en Espagne, contre un gouvernement national considéré comme illégal et usurpant le pouvoir légitime ${ }^{14}$.

Les Français, même s'ils furent les premiers à expérimenter la terrible nouveauté, continuèrent longtemps à désigner les combattants irréguliers espagnols comme des brigands, bandits, ou malfaiteurs ${ }^{15}$, comme en témoigne la correspondance du comte de La Forest, ambassadeur de France à Madrid, qui, en juillet 1808, se plaignait déjà de la difficulté des communications postales.

«Mais, [...] les lettres, les courriers sont toujours arrêtés dans un point quelconque, sur les routes mêmes où il $\mathrm{y}$ a une chaîne de postes militaires. Ce sont des brigands, des voleurs, des déserteurs [...] qui réussissent d'un côté ou de l'autre à couper les communications. $»^{16}$

«J'ai déjà eu occasion de dire [...] que le zèle des paysans, les déserteurs [...], les partis de vagabonds armés, laissent peu de chances non seulement aux courriers, mais même aux espions. $»^{17}$

5 Il faut attendre 1812 pour trouver la première trace en français de l'emploi du mot guérilla, sous la plume de Joseph de Maistre, envoyé du roi de Sardaigne à la cour du Czar. Dans une Relation pour S. M. le Roi Victor-Emmanuel, il note: "Ces paysans [...] changés en véritables guérillas et ne sachant plus que tuer, reviendront-ils des serfs dociles? $»^{18}$

6 Par contre, les autorités espagnoles au service du roi Joseph, même en ayant souvent recours aux termes bandoleros (brigands), bandidos (bandits) ou malhechores (malfaiteurs) pour désigner les civils armés qui contestaient l'occupation, utilisèrent assez tôt guerrilla dans l'acception moderne ${ }^{19}$. En anglais, le même terme fut employé pour la première fois le 8 août 1809, dans une dépêche du général Arthur Wellesley, futur duc de Wellington, au premier ministre britannique Castlereagh ${ }^{20}$. On pourra trouver ailleurs le cheminement $d u$ mot en italien et dans d'autres langues européennes ${ }^{21}$.

L'efficacité militaire de la guérilla

7 Quels furent le poids et l'importance militaire de la guérilla dans la guerre que, suivant les historiens espagnols, nous appellerons Guerre de l'Indépendance ? La discussion sur 
ce point, toujours très controversé, porte aujourd'hui essentiellement sur la question de savoir si les hommes qui, pour des raisons très diverses, participèrent à la guerre dans les bandes de guerrilleros, n'auraient pas été plus utiles à la cause espagnole en s'engageant dans les armées organisées à plusieurs reprises par le gouvernement légitime. Dans ce cas, leurs milices irrégulières auraient agi au détriment plutôt qu'à l'avantage de la cause. Le partisan de cette thèse est l'historien anglais Charles Esdaile ${ }^{22}$, qui, tout au long de sa production scientifique, n'a cessé d'affirmer que :

«... in the crucial period from the end of 1808 to the beginning of 1812, the guerrillas probably did inflict more harm on the Allied cause than they did good, for, by undermining the resistance of the regular army, they hastened the day when the French would have been able to turn overwhelming forces upon first them and then the army of the Duke of Wellington. $»^{23}$

8 Pourtant, Charles Esdaile n'appartient pas, comme cette citation pourrait incliner à le penser, au courant historiographique des historiens britanniques, qui, très nombreux, et poursuivant une tradition commencée avec l'ouvrage célèbre de W.F.P. Napier ${ }^{24}$, n'évoquent la guérilla, quand ils le font, que comme le fait de ramassis de bandits et de pillards. Tout en accordant dans ses études une large place à la guérilla et en en reconnaissant l'importance, Esdaile considère néanmoins que la contribution militaire des partidas a joué, dans l'ensemble, un rôle plus négatif que positif. En désaccord avec lui sur ce point capital $^{25}$, je ne soutiendrai pas pour autant que l'action des guérillas puisse avoir été la cause principale de la défaite des Français en Espagne, ce qui n’a été affirmé que par quelques historiens libéraux espagnols du XIXe siècle. Je reste toutefois profondément persuadé que, sans la guérilla, les Français auraient pu réussir à soumettre l'Espagne très rapidement, comme ils l'avaient fait d'autres pays d'Europe continentale. Dans cette perspective, quatre questions sensibles seront abordées : celle de l'estimation tant des pertes infligées par la guérilla aux troupes impériales que des effectifs des guerrilleros; les problèmes et les obstacles rencontrés en matière de communications ; les entraves opposées aux réquisitions de vivres et de récoltes et à la perception des impôts; la nécessité imposée aux Français d'immobiliser un grand nombre de troupes pour le contrôle du territoire, affaiblissant de ce fait la force disponible pour s'opposer aux armées alliées dans les grandes batailles rangées, qui, finalement, décidèrent de l'issue de la guerre dans la péninsule ibérique.

Le montant des pertes causées par la guérilla dans les armées impériales, aussi décisif que délicat à établir, ne saurait être que très approximatif. Le chiffre le plus élevé, certainement exagéré, a été donné par J.F.A. Le Mière de Corvey, ancien officier en Espagne, et auteur d'un important manuel sur la guerre de guérilla ${ }^{26}$.

«Cent cinquante à deux cents masses de guérillas répandues dans toute l'Espagne, avaient fait le serment de tuer chacune trente ou quarante Français par mois, cela faisait six à huit mille hommes par mois pour la totalité des bandes des guérillas. [...] Comme il y a douze mois dans l'année, nous perdions environ quatre-vingt mille hommes par an, sans avoir eu de batailles rangées : la guerre d'Espagne a duré sept ans ; c'est donc plus de cinq cents mille hommes de tués $[. ..] »^{27}$

10 Le général Bigarré, aide de camp de Joseph Bonaparte, indique quant à lui dans ses Mémoires :

«Je dois pourtant avouer que les guérillas ont fait beaucoup plus de mal aux troupes françaises que les armées régulières pendant la durée de la guerre d'Espagne; il est reconnu qu'elles n'assassinaient pas moins de cent hommes par jour. Ainsi, pendant l'espace de cinq années, elles ont tué 180000 Français sans 
avoir perdu plus de vingt-cinq mille hommes, car il était rare que ces bandes indisciplinées se battissent en rase campagne sans être plus de cinq contre un. ${ }^{28}$

Jean Sarramon, qui a longtemps travaillé à une grande et très minutieuse histoire de la guerre en Espagne, hélas encore largement inédite ${ }^{29}$, a produit un tableau détaillé des pertes françaises causées par la seule guérilla entre mai 1811 et la fin juin $1812^{30}$. Le montant final, calculé de façon très prudente, s'élève à 15888 hommes, soit moins de la moitié des 36000 pertes annuelles annoncées par Bigarré et Marbot $^{31}$; on est évidemment bien loin des calculs de Le Mière, mais, si l'on étend ce bilan d'une année aux cinq années de guerre, on approche de 80000 hommes. Il s'agit là de pertes bien plus importantes que celles causées par les armées régulières espagnoles, et presque doubles de celles provoquées par les troupes luso-britanniques de Wellington (45 000). Cette estimation globale se trouve confirmée par l'ouvrage à paraître de Ronald Fraser sur la participation populaire espagnole à la guerre ${ }^{32}$. En utilisant les données recueillies sur les pertes impériales en Navarre ${ }^{33}$, et en y ajoutant celles du reste de l'Espagne, Fraser parvient à une moyenne annuelle de 18000 hommes, soit un total de 90000 pour la durée entière de la guerre. Il ne s'agit là que des pertes dues à la guérilla. L'ensemble des pertes impériales en Espagne reste à établir : les estimations varient entre un minimum de 240000 et un maximum de 600000 hommes. Si l'estimation de Rory Muir montant à 300000 hommes $^{34}$ paraît acceptable, l'importance du rôle tenu par la guérilla semble indéniable.

Mais à combien pouvait s'élever le nombre de ceux qui ont pu imposer un si lourd tribut à la puissante machine de guerre napoléonienne? Selon mes propres hypothèses, intégrant la statistique établie par Nicolas Horta Rodríguez ${ }^{35}$, le nombre des engagés dans la guérilla en Espagne pourrait s'élever à un maximum de 65000 hommes $^{36}$, ce qui dépasse de beaucoup celui de 50000 indiqué par Arteche y $\mathrm{Moro}^{37}$, mais semble toutefois raisonnable, si l'on tient compte de la nécessité de distinguer entre les guerrilleros pour ainsi dire "permanents» et les "occasionnels", entre ceux qui pratiquèrent la guérilla pendant toute la durée de la guerre, et ceux qui, par ailleurs, ne prirent part aux combats qu'en une seule occasion et pendant un temps très réduit, ne dépassant parfois pas deux ou trois jours. Cette estimation recoupe celle donnée par Ronald Fraser. L'historien anglais, au terme de longues et difficiles recherches dans l'Espagne entière, a pu établir plusieurs bases de données sur la guérilla. L'une d'elles porte sur les partidas existant en 1811 et sur leurs effectifs, ainsi que sur la répartition entre soldats à pied et à cheval ${ }^{38}$.

Partidas de guérilla en 1811

\begin{tabular}{|c|c|c|c|c|c|}
\hline & $\begin{array}{l}\text { Grandes } \\
(>1000)\end{array}$ & $\begin{array}{l}\text { Moyennes } \\
(250-999)\end{array}$ & $\begin{array}{l}\text { PETITES } \\
(<O U=249)\end{array}$ & $\begin{array}{l}\text { Catégorie } \\
\text { inconnue }\end{array}$ & Total général \\
\hline $\begin{array}{l}\text { Nombre } \\
\& \\
\text { Dimension } \\
\text { moyenne }\end{array}$ & $\begin{array}{l}16 \\
2977,5\end{array}$ & $\begin{array}{l}11 \\
493,5\end{array}$ & $\begin{array}{l}29 \\
84,9\end{array}$ & $\begin{array}{l}56 \\
\text { nd }\end{array}$ & 112 \\
\hline \begin{tabular}{|l} 
TOTAL DES \\
gUERRILLEROS \\
PAR CATÉgORIE
\end{tabular} & 47640 & 5429 & 2462 & nd & $>55531$ \\
\hline
\end{tabular}




\begin{tabular}{|l|l||l|l|l|l|}
\hline $\begin{array}{l}\text { Total à pied } \\
\text { \& } \\
\text { moyenne/bande }\end{array}$ & $\begin{array}{l}41250 \\
2578,1\end{array}$ & $\begin{array}{l}4069 \\
369,9\end{array}$ & $\begin{array}{l}1148 \\
39,6\end{array}$ & nd & 46467 \\
\hline $\begin{array}{l}\text { Total à cheval } \\
\text { \& } \\
\text { moyenne/bande }\end{array}$ & $\begin{array}{l}6390 \\
388,2\end{array}$ & 1360 & 1314 & nd & 9064 \\
\hline \hline $\begin{array}{l}\text { \% à pied } \\
\text { \& } \\
\text { \% à cheval }\end{array}$ & $\begin{array}{l}86,6 \\
13,4\end{array}$ & 74,9 & 46,6 & nd & 83,7 \\
\hline
\end{tabular}

Source: Ronald FRASER, Database Guerrillas/Numbers. L'auteur remercie Paloma Botella, universitaire spécialiste de mathématique et de statistique, pour son travail de calcul et d'analyse des effectifs des partidas.

13 En suivant Ronald Fraser, au total de 55531 guerrilleros il convient d'ajouter ceux des 56 partidas dont les effectifs restent indéterminés. Il propose de leur attribuer les mêmes contingents qu'à ceux des petites bandes, soit 84,9 hommes chacune, ce qui établirait le total général à 60285 combattants, sans compter les guerrilleros de Catalogne et de Galice. On peut raisonnablement admettre que les partidas de ces deux régions pouvaient rassembler un effectif de 5000 hommes, formant l'écart entre les calculs de Fraser et les miens. Il faut souligner qu'à l'été 1811, les quatre armées espagnoles subsistantes ou reconstituées après les défaites successives des années précédentes ne comptant guère plus de 70000 hommes, les guerrilleros formaient ainsi une force antifrançaise presqu'égale. Ils présentaient en outre l'avantage d'être dispersés sur le territoire d'une façon assez homogène et de jouir d'une très grande mobilité, leur permettant de renoncer au combat s'ils se trouvaient en position d'infériorité.

14 Sur la composition socio-professionnelle des guerrilleros, l'on ne dispose jusqu'à présent que de l'ébauche d'étude de Nicolas Horta Rodríguez ${ }^{39}$, mais des avancées importantes sont attendues aussi bien du côté de la prochaine publication de Ronald Fraser ${ }^{40}$, que des recherches en cours de Charles Esdaile et Leonor Hernández Enviz, visant à la constitution d'une vaste base de données incluant la totalité des guerrilleros pour lesquels on détient au moins un témoignage documentaire.

Comme le soulignait l'ambassadeur de France à Madrid, les problèmes de communication à l'intérieur du territoire espagnol ou avec la France se posent dès 1808. Les attaques contre tous les types de messagers avaient commencé avant même le Dos de Mayo ; il est bien connu que l'Empecinado initia son activité contre les impériaux en avril 1808 comme caza-correos (chasse-courrier); en février de cette même année, la guérilla interceptait une lettre à Guadalajara ${ }^{41}$. Couper ou rendre difficiles les communications postales, mais aussi le passage des personnes et des marchandises d'un lieu à l'autre de l'Espagne, fut, dès le début de la résistance anti-napoléonienne, une des tâches prioritaires confiées à la guérilla. Les Prevenciones, texte diffusé par la Junte de Séville le 6 juin 1808, donnent des instructions aux provinces sur les moyens de défense à utiliser contre l'invasion française, et précisent déjà très clairement qu'il conviendra d'entreprendre :

" una guerra de partidas, de embarazos de consumir los Exército enemigos por falta de víveres, de cortar Puentes, hacer cortaduras y demas en los puntos que convenga, y otros medios semejantes. Convida á ello la situacion de España, sus muchos montes y desfiladeros, 
que ofrecen estos, sus Rios y Arroyos, la colocacion misma de las Provincias para hacer esta guerra con felicidad. ${ }^{42}$

Communications et transports pour le ravitaillement des armées, des villes et des garnisons, furent sans doute les points faibles du système d'occupation impérial en Espagne. La documentation à ce propos est accablante. Nombreux sont les hauts fonctionnaires du régime joséphin, les juges en particulier, qui demandent au ministre de la Justice l'autorisation de surseoir à la prise de possession de leur poste à cause de "la notoriedad de estar ocupados los caminos por insurgentes " ${ }^{43}$ et de la difficulté d'avoir la "seguridad de escolta ${ }^{44}$; leurs lettres portent fréquemment la mention «duplicado » ou "triplicado", car l'expédition de plusieurs exemplaires d'un même courrier par des voies différentes cherchait à obtenir qu'au moins l'un d'eux parvienne à destination, même avec un retard considérable ${ }^{45}$, comme il ressort d'une lettre, envoyée de Pampelune le 24 juillet 1809 par le général d'Agoult, gouverneur militaire de Navarre, au ministre de la Justice, qui affirme ne l'avoir reçue à Madrid que le 28 novembre suivant ${ }^{46}$ ! Il est clair que toute l'Espagne joséphine souffrait gravement de ces retards perpétuels imposés aux communications en tous genres, comme au fonctionnement quotidien de l'État et à la vie des particuliers, à quoi s'ajoutaient les difficultés des autorités militaires, sollicitées de toutes parts de fournir des escortes, indépendamment de toute considération de distance:

« Ex. mo Señor: Sin embargo de la proximidad de este Pueblo á esa Corte, para el Correo ès lo mismo que si estubiera ciento y mas leguas. Rara vez llega à tiempo: algunas le roban y muchas se detiene una semana ò mas en Alcalà, como ha sucedido ahora; siendo toda esta detencion desde dicho Pueblo acà, que son quatro leguas cortas. La excusa que dà el Conductor és que los guerrillos le interceptan y amenazan de muerte, y ni aqui, ni en Alcalà pueden ò quieren facilitar escolta los Comandantes Militares. ${ }^{47}$

Quoique les pertes infligées aux troupes impériales aient été importantes, mon opinion est que le succès majeur de la guérilla a été d'empêcher le ravitaillement régulier des armées d'occupation, soit en faisant obstruction à la libre circulation des convois, soit par une opposition opiniâtre aux exactions et aux réquisitions de récoltes, denrées alimentaires et bétail, dont les paysans étaient les principales victimes. Les impériaux devaient nourrir des effectifs compris entre 300000 et 350000 hommes, maximum atteint pendant l'été 1811. Les armées espagnoles oscillaient autour de 70000 à 100000 hommes, auxquels il convient d'ajouter les effectifs de la guérilla et ceux de l'armée anglaise, lorsqu'elle se trouvait en Espagne. Près de 700000 combattants devaient ainsi pouvoir se nourrir sur une terre où la population, paysannerie en tête, vivait difficilement, même en temps de paix. Selon la devise napoléonienne, la guerre devait alimenter la guerre; ce principe fut en effet appliqué sans trop de problèmes partout en Europe, mais pas en Espagne, où les paysans, même forcés à collaborer avec l'occupant, savaient bien qu'ensuite les guerrilleros viendraient exiger une contribution équivalente à celle qu'on avait dû céder à l'ennemi ${ }^{48}$. De plus, en offrant à la guérilla "otro auxilio mas que el de la raciòn de guerrá » (une autre aide à ajouter à la ration de guerre), ils seraient victimes d'un arrêté royal les obligeant à « mantener á su costa en raciones y sueldo, al destacamento de tropas que se le embiará $»^{49}$. Se trouvant ainsi placée entre le marteau et l'enclume, la population rurale fit le choix du moindre mal et participa à la guerre pour sa survie. Autant que possible, les paysans se rangèrent du côté de la guérilla, qui pouvait leur offrir une protection contre les troupes impériales et parfois aussi, en faveur des paysans pauvres, de l'argent ou de la nourriture pris à l'ennemi ou à quelques malheureux riches propriétaires suspectés d'être des afrancesados ${ }^{50}$. Les attaques des bandes de guerrilleros contre les colonnes militaires qui 
convoyaient pour le ravitaillement les troupeaux de moutons, de bœufs, et les chars de grains, enlevèrent enfin aux impériaux une portion considérable de leurs moyens de subsistance. On peut mesurer le succès de la guérilla au taux d'hospitalisation des troupes impériales en Espagne, 20 \% plus élevé que dans le reste de l'Europe, en raison de la sous-alimentation.

Des difficultés analogues se présentaient à propos des lourds impôts réclamés par les militaires dans les territoires occupés, du plus petit hameau jusqu'à la grande ville. Dans les campagnes surtout, les prescriptions législatives restèrent souvent lettre morte, particulièrement dès que la force des guerrilleros augmenta sensiblement, à partir des derniers mois de 1811. De toute façon, une fois l'impôt acquitté, le problème demeurait d'en faire parvenir le produit à sa destination finale, sans qu'il ne tombe aux mains des bandes qui infestaient les chemins. Cette importante fonction de la guérilla avait déjà été remarquée et signalée par l'historien anglais Charles Oman :

«The activity of the guerrilleros did not merely constitute a military danger for King Joseph.

It affected him in another, and equally vexatious, fashion, by cutting off nearly all his sources of revenue. While the open country was in the hands of the insurgents, he could raise neither imposts nor requisitions from it. The only regular income that he could procure during the later months of 1809 was that which came in from the local taxes of Madrid, and the few other large towns of which he was in secure possession. [...] The King could not command a quarter of the sum which he required to pay the ordinary expenses of government. $1^{51}$

La bataille pour les vivres et le numéraire se solda donc par une cuisante défaite française. C'est là, à mon sens, l'une des principales raisons de l'issue finale des combats.

Un autre résultat d'importance obtenu par la guérilla pendant la guerre fut de mobiliser une quantité considérable de troupes impériales dans des fonctions de police, de maintien de l'ordre et de contre-guérilla, empêchant ainsi les généraux napoléoniens d'exploiter avantageusement leur supériorité numérique sur les armées alliées, luso-britanniques dans un premier temps, hispano-luso-britanniques ensuite. Dès le début des hostilités, les effectifs impériaux en Espagne ne cessèrent d'augmenter, passant de 100000 hommes à l'automne 1807 à 350000 en juillet 1811. Ce fut seulement à la fin de cette année, qu'en préparation de la campagne de Russie, les troupes furent réduites de 70000 hommes $^{52}$. Malgré cette imposante supériorité, le maréchal Masséna ne put compter pour la campagne du Portugal que sur 65000 hommes, quand, selon le même Masséna, les impériaux disposaient en Espagne de 406348 hommes $^{53}$; l'Armée du Midi, commandée par le maréchal Soult, se composait en théorie au 1er septembre 1811 de 72000 hommes et des 4800 recrues de la garnison de Badajoz, mais ne pouvait pas en réalité aligner plus de 37000 soldats pour entrer en campagne, les autres étant, soit malades (8 500), soit, comme l'avait bien vu A.-L. Grasset dans son étude classique sur Málaga, dispersés dans de multiples petites garnisons, ou enrôlés dans les colonnes mobiles lancées à la poursuite des guerrilleros ${ }^{54}$.

«Cette équipée fatigante et coûteuse et des centaines d'autres semblables prouvaient surabondamment la difficulté de réduire par les armes les bandes insurgées. Pendant plus d'un an, les troupes de Malaga vont se consacrer, sous un ciel de feu, à cette tâche surhumaine qui les épuisera et ruinera leurs effectifs. $»^{55}$

21 Comme on l'a déjà évoqué, les impériaux devaient aussi diviser leurs forces en de nombreux détachements pour acheminer les convois ou, pire encore, tant est flagrante la disproportion entre l'objectif à atteindre et les moyens mis en œuvre pour y 
parvenir, pour escorter ne fût-ce qu'un seul messager à cheval. Les témoignages à ce propos abondent pendant toute la durée de la guerre:

«J'avais quitté Bayonne le 11 mai [1808]... l'insurrection s'organisait de toutes parts. [...] On m'escortait d'un poste à l'autre, ce qui ne m'empêcha point d'être attaqué plusieurs fois. $»^{56}$

«À cette époque [1812], on ne voyageait plus en Espagne, une fois sorti d'Andalousie, qu'avec trois ou quatre cents hommes d'escorte et quelquefois plus; encore n'était-on pas sûr d'arriver sans obstacles à sa destination; j'étais payé pour bien prendre mes précautions, d'après le combat très vif que j'avais eu à soutenir près d'Olmedo, en $1810[\ldots] \|^{57}$

En 1812 en Navarre, les escortes de messagers pouvaient mobiliser jusqu'à 600 hommes ${ }^{58}$. À la fin de 1811, du littoral méditerranéen jusqu'à Oviedo, 90000 hommes étaient requis pour protéger des attaques de la guérilla les communications avec la France ${ }^{59}$. C'est la raison pour laquelle les impériaux ne purent réunir que 46138 hommes à la bataille de Talavera, en $1809^{60}, 49634$ à celle de Salamanque, en $1812^{61}$, et seulement 62131 à la bataille de Vitoria, qui décida en 1813 du sort de la guerre ${ }^{62}$. De leur côté, les alliés purent lancer sur le terrain 55634 hommes à Talavera (20 641 Anglais et 34993 Espagnols); 51937 à Salamanque (30 578 Anglais, 17999 Portugais, 3 360 Espagnols); et plus de 101000 à Vitoria (45 102 Anglais, 27989 Portugais, 28347 Espagnols). Au moment de ce dernier engagement, les impériaux avaient plus de 70000 hommes des trois armées du Nord, de Catalogne, et du Maréchal Suchet à Valence, retenus dans leurs régions respectives pour le contrôle du territoire et la répression de la guérilla. J'espère avoir suffisamment illustré les raisons de mon désaccord avec Charles Esdaile.

En guise de conclusion, je présenterai quelques considérations générales sur le rôle et l'importance de la guérilla dans la guerre, même au-delà des pourtant considérables aspects militaires.

Les troupes impériales arrivèrent en Espagne en ayant gagné toutes les guerres entreprises jusque-là contre les armées les plus aguerries et chevronnées d'Europe, et, après avoir établi leur supériorité par une longue série de victoires écrasantes, crurent pouvoir se cantonner dans la routine quotidienne de l'occupation, comme elles l'avaient fait sans problèmes en Allemagne, en Europe centrale ou en Italie, avant la création d'un système d'États satellites de la France. Mais l'illusion fut de courte durée, le rêve se muant très vite en un horrible cauchemar, dans lequel chaque endroit du pays cachait un poignard prêt à frapper, où un verre de vin offert avec le sourire était très souvent empoisonné. Dans un ouvrage récent, Alan Forrest est net à cet égard :

"If service in Italy seemed threatening, Spain was even worse. The war in the Peninsula enjoyed a particularly fearsome reputation, in part -but only in part-as a consequence of the army's intensive propaganda against the Spaniards. Napoleonic soldiers seem to have shared a horror of fighting there, especially after experience of other theatres of war, notably Germany and Central Europe. Spain was widely believed to be a savage and inhospitable country where French soldiers were left to die by callous villagers set on vengeance, where local people refused to sell them foodstuffs, and where they were virtually prisoners in their camps because of the activity of local "brigands". $"^{63}$

Les soldats impériaux, les jeunes recrues comme les grognards les plus expérimentés, éprouvaient à l'égard de l'Espagne une espèce de crainte révérencielle qui affectait leurs capacités militaires et encourageait la désertion, surtout chez les alliés, de même que l'insoumission en France ${ }^{64}$. D'après Charles Esdaile, les déserteurs français auraient, en 1811, dépassé le nombre de $50000^{65}$. La guérilla a également apporté un important 
soutien psychologique à la population espagnole, surtout pendant les années 1810 et 1811, lorsque les nouvelles des champs de bataille ne faisaient état que de terribles défaites, que la confiance envers les armées ne cessait de faiblir, et qu'il semblait que le tyran de l'Europe allait triompher une fois de plus. Les guerrilleros jouissaient du soutien populaire, qui s'exprimait aussi bien par l'appui matériel apporté (nourriture, hébergement...) qu'à travers les renseignements sur les mouvements de l'ennemi, ou les fausses informations dispensées aux impériaux. Personne ne songe à nier qu'il y ait eu des scènes de brigandage, de pillage, de violence, atroces parfois, imputables à des hommes ou à des bandes qui se réclamaient de la guérilla. On ne peut pas davantage nier que la population et les authentiques guerrilleros dénonçaient en général ces malfaiteurs, même aux autorités joséphines ou impériales, ou bien exerçaient directement sur eux une justice sommaire. Sans vouloir mythifier la guérilla, ni en exagérer l'importance, on doit de toute façon admettre que son existence et sa présence active, pendant les longues et difficiles années de la guerre, exercèrent un poids essentiel sur l'issue du conflit.

\section{NOTES}

1.Pour une vision plus ample des thèmes abordés ici, je me permets de renvoyer à mes articles «Spagna 1808: la genesi della guerriglia moderna. 2. Fenomenologia della guerriglia spagnola e suoi riflessi internazionali ", Spagna contemporanea, $n^{\circ} 20,2001$, pp. 73-167; « La guerilla en la Guerra de la Independencia : ayuda imprescindible para la victoria o estorbo grave e inoportuno? ", Primeras Jornadas de Estudio sobre la Guerra de la Independencia en Málaga y su provincia (1808-1814), Actas del Colloquio, octubre de 2002 (à paraître).

2.Voir l'essai de T. Barata, $O$ desenvolvimento doutrinário e a importância crescente da guerra subversiva nos dois últimos séculos, Lisboa, Acta dos Colóquios Internacionais, 1990-1991-1992.

3.Cité par W. Hahlweg, Guerrilla. Krieg ohne Fronten, Stuttgart-Berlin-Köln-Mainz, Kohlhammer, 1968. Trad. italienne (utilisée ici) Storia della guerriglia. Tattica e strategia della guerra senza fronti, Milano, Feltrinelli, 1973, p. 21. La définition est de V.-R. Wolf, R.W. Günter, G. Moritz, Der Verdeckte Kampf, Bonn, Offene Worte, 1965, p. 1.

4.Trad. récente de ce texte en italien, Thomas Edward Lawrence, Guerrilla-Guerriglia, Roma, Stampa Alternativa, 2002. Cf André Guillaume, Lawrence d'Arabie, Paris, Fayard, 2000. La doctrine de Lawrence sur la guérilla est résumée dans le chapitre 33 de son ouvrage Seven Pillars of Wisdom. A Triumph, imprimé une première fois à titre privé en 1926, puis en 1935 à Londres.

5.Sebastián de Covarrubias Orozco, Tesoro de la Lengua Castellana o Española, Madrid, Luis Sánchez, 1611 ; réimpr. par F. C. R. Maldonado, Madrid, Castalia, 1995, p. 613, entrée guerra: cuando entre particulares hay pendencia y enemistad formada, que acuden unos a una parte y otros a otra; pero éstas castigan los príncipes de las repúblicas severamente. 
6.À propos du succès des traités français en Espagne, voir M.-R. Garcia Hurtado, Traduciendo la guerra. Influencias extranjeras y recepción de las obras militares francesas en la España del siglo XVIII, A Coruña, Universidade da Coruña, 1999, ainsi que le compte rendu que j'en ai fait : « La guerra « alla francese » nel XVIII secolo e la sua fortuna in Spagna ", Spagna contemporanea, $\mathrm{n}^{\circ} 17,2000$, pp. 161-163.

7.Sur la genèse et le développement de la petite guerre, et plus généralement sur la guerre irrégulière, je renvoie à mon article « Spagna 1808: la genesi della guerriglia moderna. 1. Guerra irregolare, petite guerre, guerrilla ", Spagna contemporanea, $\mathrm{n}^{\circ} 18$, 2000, pp. 9-31.

8.Publié à Paris en 1756, l'ouvrage de Philippe Augustin Thomas de Grandmaison fut rapidement traduit en allemand (Frankfurt et Leipzig, 1758), puis en espagnol (1780), à nouveau en allemand (Wien, 1785) et en espagnol (1794). On continua de le rééditer tout au long du XIXe siècle.

9.Trad. du capitaine Víctor Amadeo María Caballero, Valence, Salvador Fauli, avec ajouts de considérations personnelles et de notas de los más extraordinarios sucesos acaecidos en la guerra à las Tropas Ligeras. Voir M.-R. Garcia Hurtado, op. cit., pp. 103-104. 10.Pour l'histoire et la fortune d'un autre mot étroitement lié à la conception moderne de la guérilla, «partisan », voir l'art. cit. supra, note 7.

11.J. Gomez De Arteche, Guerra de la Independencia: historia militar de España de1808 a 1814, 14 vol., Madrid, Depósito de la Guerra, 1866-1903, II, pp. 692-696 (ouvrage en cours de réédition à Valencia, chez Simtac).

12.Apuntes de la vida y hechos militares del brigadier Don Juan Martín Díez El Empecinado por un admirador de ellos, Madrid, s.e., 1814, p. 6.

13.Par exemple Natalio Rivas Santiago, El alcalde de Otivar, héroe en la Guerra de la Independencia, Madrid, s.e., 1940, pp. 28 et 30. On trouve également Partida de guerrilla au sens de « formation d'infanterie légère » dans F. Casamayor, Diario de los sitios de Zaragoza, (rédigé en 1808), éd. par H. Lafoz Rabaza, Zaragoza, Comuniter, 2000, pp. 49 et 65, et dans le bulletin du Général Castaños déjà cité.

14.Pour un usage très récent du terme, voir Le Monde, 9 juillet 2003: « Pour les spécialistes militaires, les combats ont pris maintenant la forme d'une guérilla classique. Le faible attaque le fort à l'endroit et au moment qu'il choisit et se fond ensuite dans la population. Plus la réponse est brutale, plus les civils ont tendance à considérer les assaillants avec bienveillance ».

15.Jean-René Aymes, « La guerrilla española (1808-1814) en la literatura testimonial francesa », dans P. Molas Ribalta (éd.), La España de Carlos IV, Madrid, Tabapress, 1991. 16.G. de Grandmaison (éd.), Correspondance du Comte de La Forest Ambassadeur de France en Espagne 1808-1813, 7 vol., Paris, Picard, 1905-1913, I (1905), Avril 1808-janvier 1809, p. 147 (6 juillet 1808).

17.Ibid., p. 154 (10 juillet).

18.Joseph de Maistre, Correspondance, 6 vol., Lyon, Vitte et Perrussell, 1884, IV, p. 282. Les Oeuvres complètes: contenant ses oeuvres posthumes et toute sa correspondance inédite de J. de Maistre ont été réimprimées par G. Olms, Hildesheim, 1984.

19.Par exemple dans un document signé par le ministre de Police, Pablo Arribas, adressé au Roi le 24 janvier 1810. Voir Archivo General de Simancas (désormais AGS), sección Gracia y Justicia (désormais GyJ), legajo (désormais leg.) 1076, sin foliar : «... con aquellos que habiendo sido seducidos han formado parte de dichas quadrillas de guerrilla...» (« ...avec ceux qui, séduits par la guérilla, ont voulu faire partie de ses bandes... »). 
20.A. Wellesley, The Dispatches of Field Marshal the Duke of Wellington During His Various Campaigns in India, Denmark, Portugal, Spain, the Low Countries, and France, from 1779 to 1815, éd. par le Lt-Col. Gurwood, 12 vol., London, John Murray, 1834-1838, V, pp. 9 et 12. Le général anglais emploie le terme dans l'acception moderne.

21.Cf. art. cité, supra, note 7.

22.Esdaile est le seul grand spécialiste britannique contemporain de la Peninsular War. La liste de ses publications est remarquable: The Spanish Army in the Peninsular War, Manchester, Manchester University Press, 1988; « Heroes or Villains. The Spanish Guerrillas in the Peninsular War ", History today, 4, 1988, pp. 29-35; The Duke of Wellington and the Command of the Spanish Army, 1812-1814, London, Macmillan, 1990; "The problem of the Spanish guerrillas », dans A. Berkeley (éd.), New Lights on the Peninsular War: International Congress on the Iberian Peninsula, 1780-1840, Lisbon, The British Historical Society of Portugal, 1991; The Wars of Napoleon, London and New York, Longman, 1995; « Rebeldía, reticencia y resistencia: el caso gallego de 1808 », Trienio, n 35, 2000, pp. 57-80; Spain in the Liberal Age. From Constitution to Civil War, 1808-1939, Oxford, Blackwell, 2000; The Peninsular War. A New History, London, Allen Lane, 2002. Le dernier livre d'Esdaile porte précisément sur le thème en question ici (Fighting Napoleon. Guerrillas, Bandits and Adventurers in Spain, 1808-1814, New Haven-Londres, Yale Univ. Press, 2004).

23. Charles Esdaile, « Heroes or Villains revisited: fresh thoughts on la guerrilla », Il Seminario Internacional sobre la Guerra de la Independencia, Madrid, 24-26 octobre 1994, Madrid, Ministerio de Defensa, 1996, pp. 191-210. Citation pp. 209-210.

24.William Francis Patrick Napier, History of the War in the Peninsula and in the South of France from the year 1807 to the year 1814, 6 vol., London, Warne \& Co., 1890-1892 (1ere éd., Londres, 1828-1840).

25.Une première réponse aux positions soutenues par Esdaile est contenue dans la communication présentée au colloque de Màlaga, citée supra.

26.Jean Frédéric Auguste Le Miere de Corvey, Des partisans et des corps irréguliers ou Manière d'employer avec avantage les troupes légères, quelle que soit leur dénomination: Partisans, Voltigeurs Compagnies-Franches, Guérillas, et généralement toute espèce de Corps irréguliers, contre des Armées disciplinées. Ouvrage utile dans les guerres régulières, et indispensable dans le cas d'une invasion étrangère, Paris, Anselin et Pochard, 1823. 27.Ibid., pp. 101-102.

28.Auguste Bigarré, Mémoires du général Bigarré, Aide de camp du Roi Joseph, 1775-1813, Paris, Kolb, 1893, p. 278.

29.Jean Sarramon a consacré douze volumes à la description détaillée de toutes les actions militaires, jusqu'à la moindre, pendant les vingt-six mois de la période mai 1811- juin 1813. Deux volumes seulement ont été publiés: La bataille des Arapiles (22 Juillet 1812), Toulouse, Publications de l'Université Toulouse-Le Mirail, 1978; La bataille de Vitoria. La fin de l'aventure napoléonienne en Espagne, Paris, Bailly, 1985. L'ouvrage de Sarramon peut être consulté en microfilm aux Archives de la Guerre au Château de Vincennes et à l'Instituto de Historia y Cultura Militar de Madrid. Du même auteur, Napoléon et les Pyrénées. Les chasseurs des montagnes et la couverture de la frontière 1808-1814, Selgues, Le Lézard, 1992.

30.Jean Sarramon, La bataille des Arapiles (...), op. cit., p. 431.

31.J.B.A.M. Marbot, Mémoires 1799-1815, 3 vol., Paris, Plon, 1891, II, p. 484. Marbot pense en effet que « dans les six années qui se sont écoulées depuis le commencement de 1808 jusqu'à la fin de 1813, les Français ont perdu dans la péninsule ibérique 200000 hommes tués ou morts 
dans les hôpitaux, auxquels il faut ajouter les 60000 perdus par nos alliés des diverses nations ". Même en déduisant les pertes subies au Portugal, on est proche de l'estimation de Bigarré.

32.Je tiens à remercier vivement Ronald Fraser, ainsi que la maison d'édition Faber \& Faber, de m'avoir autorisé à prendre connaissance d'une partie du manuscrit à paraitre sous le titre To Die in Spain. Popular Resistance in the Peninsular War.

33.J. L. Tone, La guerrilla española y la derrota de Napoleón, Madrid, Alianza, 1999, p. 321. Éd. originale, The Fatal Knot. The Guerrilla War in Navarre and the Defeat of Napoleon in Spain, Chapel Hill et Londres, The University of North-Carolina Press, 1994. L'édition espagnole contient une traduction partielle de l'édition originale, à laquelle de nouveaux chapitres ont été ajoutés. Les pertes en Navarre données par Tone et reprises par Fraser, ne sont que celles causées par les deux Mina (Javier et Francisco) de 1810 à la fin de la guerre : 17103 tués ou faits prisonniers (358 par Javier Mina, 16745 par Francisco Espoz).

34.Rory Muir, Britain and the Defeat of Napoleon 1807-1815, New Haven and London, Yale University Press, 1996, p. 421, n. 9 et 11.

35.Nicolas Horta Rodriguez, « Sociología del movimiento guerrillero ", dans M. Hernandez Sanchez-Barba \& M. Alonso Baquer (dir.), Historia social de las fuerzas armadas españolas, 8 vol., Madrid, Alhambra, 1986, II, Revolución nacional e independencia, p. 511. 36.V. Scotti Douglas, Spagna 1808 (...), art. cité, pp. 94-96.

37.José de Arteche y Moro, Guerra de la Independencia. Historia militar de España de 1808 a 1814, 14 vol., Madrid, Déposito de la Guerra, 1886-1903, II, p. 125.

38.Données aimablement communiquées par l'auteur. Cf. note 32 .

39.Cf. note 35 .

40.Elle contient de nombreuses données statistiques sur la provenance sociale des guerrilleros et sur leur profession d'avant-guerre.

41.Dans l'Archivo Histórico Nacional de Madrid (désormais AHNM), Estado, leg. 3003, 3068, 3069, 3070, 3078, 3091, 3096, 3100, 3108, 3111, 3112, 3116, 3119 et 3130, Fraser a dénombré 1626 lettres interceptées. D'autres se trouvent dans le 84 legajos (Estado), avec l'indication Papeles de la Junta Central Suprema Gubernativa del Reino y del del Consejo de Regencia (numérotés 1-84).

42." une guerre de bandes, d'obstacles pour miner les armées ennemies par le manque de vivres, la coupure des ponts ainsi que toutes sortes de coupures là où il soit convenable, et d'autres moyens semblables. La situation de l'Espagne, son caractère montagneux avec ses défilés, ses fleuves et ses ruisseaux, et même la disposition de ses Provinces facilitent cette sorte de guerre, ainsi que sa réussite » (Prevenciones, promulgadas por la Junta Suprema de Gobierno de España e Indias, el 6 de junio de 1808, « Colección documental del Fraile », Archivo Histórico Militar, Madrid, vol. DCCCLXIV, p. 50).

43.« la notoriété du fait que tous les chemins sont occupés par des insurgés ».

44. « la sécurité de l'escorte» (AGS, GyJ, leg. 1078, sin foliar, lettre de Palencia du 27 octobre 1810, de Pedro Joaquín Escudero, président de la Junta Criminal Extraordinaria de Palencia, à Manuel Romero, ministre de la Justice).

45.Autres exemples cités dans mon article «L'Archivo General de Simanca, fonte misconosciuta per la storia del regno di Giuseppe Bonaparte ", Spagna contemporanea, $\mathrm{n}^{\circ}$ 7, 1995, pp. 189-194. 46.AGS, GyJ, leg. 1083, sin foliar. 
47.« Monseigneur : Malgré la proximité de ce village vis-à-vis de la Cour, pour le courrier c'est la même chose que s'il se trouvait à plus de cent lieues. Il n'arrive presque jamais à temps : quelquefois il est volé et d'autres il est retenu une ou plusieurs semaines à Alcalá, comme cela vient d'arriver récemment, tandis que ce village se trouve seulement à quelque quatre lieues. L'excuse que donne le conducteur, c'est que les guérilleros l'ont intercepté et l'ont menacé de mort, et ici, pas davantage qu'en Alcalá, les Commandants militaires ne peuvent -ou ne veulent- offrir aucune escorte » (AGS, GyJ, leg. 1121, sin foliar, lettre de Guadalajara du 26 juin 1811, de Diego Gallardo, préfet de la ville, au ministre de la Justice).

48.Cas prévu par l'art. 16 du règlement sur le Corso terrestre, Instruccion que su Magestad se ha dignado aprobar para el corso terrestre contra los exércitos franceses, AHNM, Estado, Papeles de la Junta Central, leg. 51A, doc. 6. À propos des lois promulguées par les autorités espagnoles sur la guérilla, voir V. Scotti Douglas, « Guerrillas y autoridades patriotas en la Guerra de la Independencia ", dans AA.VV., La Guerra de la Independencia en el Valle Medio del Ebro. Segundo Curso de Verano de Tudela, Tudela, Ayuntamiento de Tudela, 2003, pp. 169-210.

49." entretenir avec leurs propres moyens - en rations et en argent - le détachement de troupes qu'on va lui envoyer » (Real Decreto du 7 août 1811, AGS, GyJ, leg. 1084, sin foliar) ; autre exemplaire de l'arrêté, leg. 1108, sin foliar.

50.Afrancesado : littéralement «francisé ». Le mot était utilisé pour identifier les partisans de Joseph Bonaparte.

51.Charles Oman, A History of the Peninsular War, Oxford, Clarendon Press, 7 vol., 1902-1930, III, p. 116.

52.David Gates, The Spanish Ulcer. A History of the Peninsular War, London, Allen \& Unwin, 1986, Appendice 2, pp. 481-530. Au 15 juillet 1811, les impériaux avaient en Espagne 354 461 hommes, dont 291414 prêts au combat, ibid., pp. 504-507.

53.AHNM, Estado, leg. 3003. Le chiffre de Masséna diffère de celui de David Gates, qui propose toutefois un effectif élevé (note précédente).

54.Alphonse-Louis Grasset, Malaga, Province française (1811-1812), Paris, Lavauzelle, 1912, pp. 20-21 et 573-577.

55.Ibid. pp. 52-53.

56.J.B.A.M. Marbot, op.cit., II, p. 45.

57.A.-A.-R. de Saint-Chamans, Mémoires du général comte de Saint-Chamans ancien aide de camp du Maréchal Soult 1802-1832, Paris, Plon Nourrit, 1896, p. 208.

58.Service Historique de l'Armée de Terre (SHAT), Paris, Château de Vincennes, C8, 387, Relation du général Abbé, 1er février 1812.

59.Basil Liddell Hart, The Strategy of Indirect Approach, London, Faber and Faber, 1941, p. 148.

60.D'après David Gates, The Spanish Ulcer (...), op. cit., pp. 490-492.

61.D'après Rory Muir, Salamanca 1812, New Haven and London, Yale University Press, 2001, Appendix II et III, pp. 240-264.

62.Jean Sarramon, La bataille de Vitoria (...), op.cit., pp. 678-687.

63. Alan Forrest, Napoleon's Men. The Soldiers of the Revolution and Empire, London-New York, Hambledon and London, 2002, pp. 123-124.

64.Id., Conscripts and Deserters. The Army and French Society during the Revolution and Empire, New York-Oxford, Oxford University Press, 1989.

65. Charles Esdaile, The Wars of Napoleon, op.cit., pp. 247-249. 


\section{RÉSUMÉS}

Après avoir exposé le changement de signification du mot espagnol guerrilla, de l'acception originelle d'« opération militaire secondaire, conduite par de petits groupes de soldats » à celle, moderne, de «lutte armée de civils contre un envahisseur/occupant et ses alliés ", le problème posé est celui de l'efficacité militaire de la guérilla antinapoléonienne. Quatre aspects en sont abordés : les pertes infligées par la guérilla aux impériaux; les difficultés de communication qu'elle provoque, ainsi que les obstacles opposés au ravitaillement des troupes; enfin, la nécessité, pour les impériaux, d'utiliser la plus grande partie de leurs forces pour contrôler le territoire, ne pouvant ainsi profiter de leur supériorité numérique dans les batailles rangées contre les armées luso-anglo-espagnoles. La guérilla fut également un moyen non négligeable de soutien psychologique pour la population espagnole, alors que les troupes impériales redoutaient d'être envoyées en Espagne, où elles arrivaient déjà minées par la crainte.

\section{The Spanish Guerrilla War against Napoleon's Army}

After discussing the change in meaning of the Spanish word guerrilla, from the original sense of "secondary military operation conducted by small groups of soldiers" to the modern one of "armed civilian struggle against an invader/occupier and their allies", the problem raised is that of the military effectiveness of the anti-Napoleonic guerrilla war. Four aspects are reviewed : the losses inflicted by the guerrillas on the Imperial army; the communication difficulties arising, including the obstacles put in the way of troop supplies; finally, the need for the Imperial army to use the greater part of their forces to control the territory, thus preventing them from making use of their numerical superiority in the order of battle against the Anglo-Luso-Spanish armies. Guerrilla warfare was also a non-negligible means of bringing psychological support to the Spanish population, while the Imperial troops dreaded the prospect of being sent to Spain, where they set foot already undermined by fear.

\section{INDEX}

Mots-clés : Espagne, communications, effectifs, guérilla, pertes, ravitaillement

\section{AUTEUR}

\section{VITTORIO SCOTTI DOUGLAS}

Università di Trieste 